\title{
The Influence of Material Parameters on SRM Grain Structure Integrity under Internal Pressure
}

\author{
Bin Chen, Wei Peng
}

\author{
Department One, Mechanical Engineering College, Shi Jia Zhuang, Hebei, P. R. China
}

\begin{abstract}
Keywords: FEM; SRM; material parameters; Von Mises strain
Abstract: The material properties play an important part in evaluating the structure integrity of the solid rocket motor gain. Based on the structure integrity analysis of perfect solid rocket motor grain, the computational models are established to assess the effects of material parameters on it. Under the internal pressure, four test groups are performed. Each test group only had one variable, while the values of other variables were fixed. Through changing the value of the variable, the relationship between the Von Mises strain of dangerous point and variables are studied. The results show that the main factors are the initial elastic modulus and the poisson's ratio of propellant.
\end{abstract}

\section{Introduction}

Based on three-dimension viscoelastic theory, the analysis of perfect solid rocket motor (SRM) structure integrity has been provided an effective technique to its design and manufacture. Liu and Li (2004) analyzed the deformation of solid propellant grains under axial acceleration load. Kong (2011) discussed the effect of geometric parameters on the structural integrity of wheel-shaped grain. Sun (2016) evaluated the structure integrity of SRM grain with impurity at different temperatures and found the influence of the impurity under the temperature loading. Besides those, the material properties (Ding, 2007; Liu, 2009) play an important role in the solid propellant grain, which cannot be eliminated. This manuscript presents describes the effects of solid rocket motor grain material parameters on structure integrity under internal pressure. These conclusions would be helpful to the design and manufacture of solid rocket motors.

\section{Finite element methods}

\subsection{Linear Viscoelastic Constitutive Equations}

For isotropic linear viscoelastic materials, the stress-strain relation can be written as incremental form (Zhu, 1997; Yang, 2004), as in equation (1).

$$
\Delta \sigma=D_{e c}\left(\Delta \varepsilon-\Delta \varepsilon^{\prime}\right)
$$

where $\Delta \sigma=\left\{\Delta \sigma_{x}, \Delta \sigma_{y}, \Delta \sigma_{z}, \Delta \tau_{x y}, \Delta \tau_{y z}, \Delta \tau_{x z}\right\}^{\mathrm{T}}$ is the incremental stress vector; $D_{e c}$ is the elasticcreep matrix, its expression is presented in the published literature (Yang, 2004); $\Delta \varepsilon=\left\{\Delta \varepsilon_{x}, \Delta \varepsilon_{y}\right.$, $\left.\Delta \varepsilon_{z}, \Delta \gamma_{x y}, \Delta \gamma_{y z}, \Delta \gamma_{x z}\right\}^{\mathrm{T}}$ is the incremental strain vector; and $\Delta \varepsilon^{\prime}=\left\{\Delta \varepsilon_{x}^{\prime}, \Delta \varepsilon_{y}^{\prime}, \Delta \varepsilon_{z}^{\prime}, \Delta \gamma_{x y}^{\prime}, \Delta \gamma_{y z}^{\prime}\right.$, $\left.\Delta \gamma^{\prime}{ }_{x z}\right\}^{\mathrm{T}}$ is the pseudo-incremental strain vector.

\subsection{Virtual work equation}

The stress-strain field of linear viscoelastic materials is analyzed by using finite element solution, and six-sided solid isoparametric element with eight nodes is adopted to mesh the motor grain. For arbitrary element, between any time incremental steps, such as $t_{k}$ to $t_{k+1}$, the incremental displacement and strain vector are written in the following form.

$$
\begin{aligned}
\Delta u_{k+1} & =N \Delta u_{i k+1} \\
\Delta \varepsilon_{k+1} & =B \Delta u_{i k+1}
\end{aligned}
$$


where $\Delta u_{i k+1}=u_{i k+1}-u_{i k}$ is the incremental displacement vector of node; $N$ is the shape function matrix; $B$ is the stress matrix, respectively.

The virtual work equation is written as in equation (4).

$$
\int_{v} \delta \varepsilon^{T} \Delta \sigma_{k+1} d v=\int_{v} \delta u^{T} \Delta P_{k+1} d v+\int_{s} \delta u^{T} \Delta F_{k+1} d s
$$

where $\delta \varepsilon^{T}$ is the virtual strain; $\delta u^{T}$ is the virtual displacement; $\Delta P_{k+1}$ is the incremental vector of body force between time step $t_{k}$ to $t_{k+1}$; and $\Delta F_{k+1}$ is the incremental vector of facial or concentrated force on the boundary.

Substituting equation (1) into equation (4), the equilibrium equations are obtained by summing the contributions of each element, as in equation (5).

$$
K \Delta u_{i k+1}=\Delta Q_{k+1}
$$

Where

$$
\begin{gathered}
K=\int_{v} B^{T} D_{e c} B d v \\
\Delta Q_{k+1}=q_{1}+q_{2}+q_{3} \\
q_{1}=\int_{v} N^{T} \Delta P_{k+1} d v \\
q_{2}=\int_{s} N^{T} \Delta F_{k+1} d s \\
q_{3}=\int_{v} B^{T} D_{e c} \Delta \varepsilon_{k+1}^{\prime} d v
\end{gathered}
$$

\subsection{Evaluation criterion of structure integrity}

As the propellant is viscoelastic material, the Von Mises strain criterion is more reasonable to evaluate the structure integrity of motor grain. The Von Mises strain criterion can be expressed as in equation (11).

$$
\varepsilon_{V} \leq \frac{\varepsilon_{V m}}{n}
$$

where $\varepsilon_{V m}$ is the critical Von Mises strain; $n$ is the safe coefficient; $\varepsilon_{V}$ is the Von Mises strain of the dangerous point of SRM grain.

Under the uniaxial loading, the relation of the critical Von Mises strain and the maximum stretch ratio can be obtained as in the following form.

$$
\varepsilon_{V m}=\frac{2}{3}(1+v) \varepsilon_{m}
$$

\section{Computational models}

\subsection{Three-dimensional finite element model}

Because the solid rocket motor is a cylindrical object, and the loading of it is also axisymmetric. One-sixteenth of an axisymmetric part of the SRM is considered for the analysis. The finite element meshes of the grain using six-sided solid element with eight nodes are shown in Figure 1. We set up 101,536 nodes and 95,251 solid elements in this model, which can ensure the accuracy of simulation analysis. According to the test data, we found that the time of internal pressure building up to peak pressure is $0.2 \mathrm{sec}$, and the value of peak pressure is $5 \mathrm{MPa}$. 


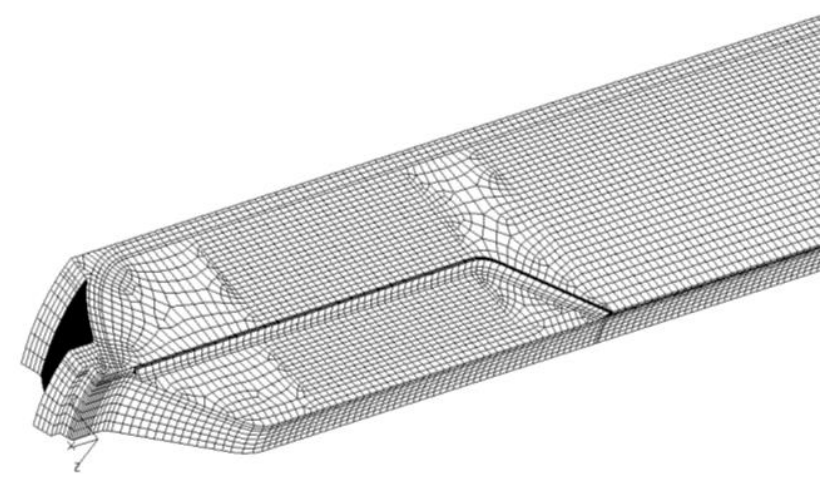

Figure 1. The three-dimensional finite element model of the motor grain.

\subsection{Material parameters}

In general, the main of SRM consists of propellant, cladding, insulation and case. In this manuscript, the influence of material parameters of the propellant and cladding is discussed by controlling the variables. So, values used for elastic modulus and poisson's ratio of the insulation and the case are fixed values, which are shown in table 1.

Table 1. Material parameters of insulation and case.

\begin{tabular}{|c|c|c|}
\hline & $\begin{array}{c}\text { Elastic modulus } \\
/ \mathrm{Mpa}\end{array}$ & Poisson's ratio \\
\hline Insulation & 30 & 0.490 \\
\hline Case & 200,000 & 0.3 \\
\hline
\end{tabular}

There are four variables, the initial elastic modulus of propellant, the poisson's ratio of propellant, the initial elastic modulus and poisson's ratio of cladding, in the trial. So, four groups of experiments were performed. In each group of experiments, values of three different variables were fixed, while the remaining one was changed according to the rules shown in the table 2 . Then, the change of the trial results was studied.

Table 2. Four groups of experiments.

\begin{tabular}{|c|c|c|c|c|}
\hline & \multicolumn{3}{|c|}{ Fixed values of three variables } & Changing rules of remaining variable \\
\hline \multirow{2}{*}{$\begin{array}{c}\text { Group } \\
1\end{array}$} & E/cladding/Mpa & $v /$ propellant & $v /$ cladding & E/propellant/Mpa \\
\cline { 2 - 5 } & 5.65 & 0.498 & 0.498 & $0.1 \mathrm{E}, 0.5 \mathrm{E}, \mathrm{E}, 2 \mathrm{E}, 4 \mathrm{E}, 6 \mathrm{E}, 8 \mathrm{E}, 10 \mathrm{E}$ (E=7.06) \\
\hline \multirow{2}{*}{$\begin{array}{c}\text { Group } \\
2\end{array}$} & E/propellant/Mpa & E/cladding/Mpa & $v /$ cladding & $v /$ propellant \\
\cline { 2 - 5 } & 7.06 & 5.65 & 0.498 & $0.49,0.492,0.494,0.496,0.498,0.499$ \\
\hline \multirow{2}{*}{$\begin{array}{c}\text { Group } \\
3\end{array}$} & E/propellant/Mpa & $v /$ propellant & $v /$ cladding & E/cladding/Mpa \\
\cline { 2 - 5 } & 7.06 & 0.498 & 0.498 & $0.1 \mathrm{E}, 0.5 \mathrm{E}, \mathrm{E}, 2 \mathrm{E}, 4 \mathrm{E}, 6 \mathrm{E}, 8 \mathrm{E}, 10 \mathrm{E}$ (E=5.65) \\
\hline $\begin{array}{c}\text { Group } \\
4\end{array}$ & E/propellant/Mpa & E/cladding/Mpa & $v /$ propellant & v/cladding \\
\cline { 2 - 5 } & 7.06 & 5.65 & 0.498 & $0.2,0.25,0.3,0.35,0.4,0.45,0.499$ \\
\hline
\end{tabular}

\section{Results and discussion}

\subsection{The effect of elastic modulus of propellant}

In the test group 1, the values of propellant and cladding poisson's ratio are both set to 0.498 . At the same time, the value of the initial elastic modulus of propellant is changed from $0.1 \mathrm{E}_{\mathrm{p}}$ to $10 \mathrm{E}_{\mathrm{p}}$. Then, the Von Mises strain field of SRM grain was calculated by finite element model. Comparing the highest value of strain, the effect of the initial elastic modulus of propellant on the dangerous point of motor grain is studied. The rule of Von Mises strain of dangerous point variation with the 
propellant elastic modulus is shown in Figure 2. When the propellant initial modulus decreased to $50 \%$ of the original value, Von Mises strain of dangerous point growth soon. In another word, for the propellant with low modulus, internal pressure is a major cause that affects its structural integrity. That is because propellant is the main component bearing the load. So when developing a new type of propellant, the propellant initial modulus should be appropriate to improve on the premise of considering other performance.

\subsection{The effect of Poisson's ratio of propellant}

In this group, the value of the poisson's ratio of cladding is set to 0.498 . Then, changing the propellant poisson's ratio $v_{\mathrm{p}}$ from 0.49 to 0.498 , and calculating the strain of dangerous point. The results are shown in Figure 3.

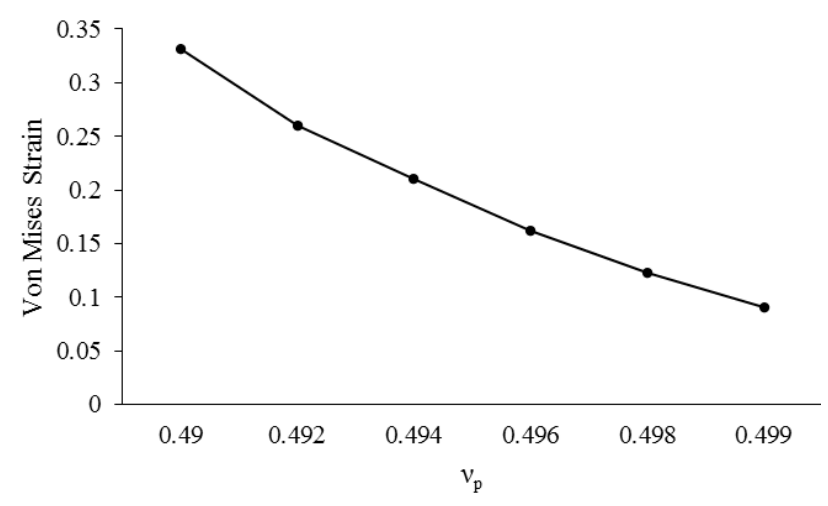

Figure 3. The Von Mises strain of dangerous point variation with the propellant poisson's ratio.

It can be seen that the effect of propellant poisson's ratio is very obvious. The Von Mises strain of the surface of the grain grooves is linear decrease approximately with the increase of propellant poisson's ratio. If the propellant poisson's ratio changes $2 \%$, the strain of dangerous point changes $75.8 \%$. The influence of poisson's ratio is much bigger than the influence of the initial modulus. Therefore, when choosing the substrate of propellant, the material with higher value of poisson's ratio should be considered preferentially.

\subsection{The effect of elastic modulus of cladding}

In group 3, the value of elastic modulus of propellant is set to $7.06 \mathrm{Mpa}$, while the value of the cladding's changes from $0.1 \mathrm{E}_{\mathrm{c}}$ to $10 \mathrm{E}_{\mathrm{c}}$. The rule of Von Mises strain of dangerous point variation with the cladding elastic modulus is shown in Fig.4. It shows that the effect of cladding initial modulus is not obvious. Note that when the initial modulus of cladding changes for 100 times, the change of the value of grain Von Mises strain is just 7.6\%. As for the reason, the cladding layer is just $1 \sim 1.5 \mathrm{~mm}$, the effect of the change of its initial modulus on propellant Von Mises is naturally small. So when choosing the material of cladding, it can pay more attention to the technical requirements to adjust the cladding modulus.

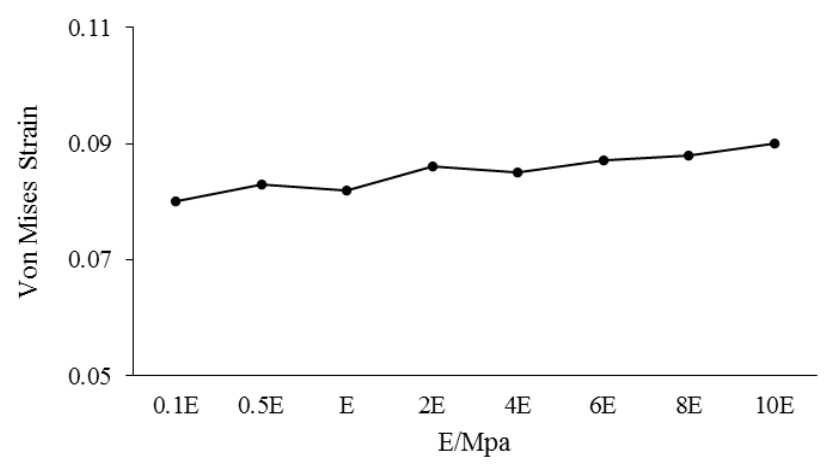

Figure 4. The Von Mises strain of dangerous point variation with the cladding modulus. 


\subsection{The effect of poisson's ratio of cladding}

In the last test group, the value of propellant poisson's ratio is set to 0.498 . Then, the cladding poisson's ratio $v_{c}$ is changed from 0.2 to 0.498 in finite element model. At the same time, the Von Mises strain of dangerous point and the highest Von Mises strain of cladding itself with the change are calculated, respectively. The rules are shown in Figure 5. The solid line is the variation of propellant Von Mises strain with the poisson's ratio of cladding, the dotted line is cladding's. It can be seen that the Von Mises strain of the propellant and cladding in dangerous point are linear decrease approximately with the increase of cladding poisson's ratio. Not only is the effect of cladding poisson's ratio on dangerous point strain of propellant big, but also the effect on strain of cladding itself is more obvious. Note that when the value of cladding poisson's ratio is smaller than 0.475 probably, the value of cladding Von Mises strain is bigger than that of dangerous point strain of propellant. Furthermore, if the value of cladding poisson's ratio drop to below 0.45 , the value of dangerous point strain of propellant will be higher than the value $16 \%$, which is the critical Von Mises strain. The RSM grain will be very dangerous in this situation. So when developing a new type of propellant, the designer is better to choose the cladding's materials that the poisson's ratio is greater than 0.49 .

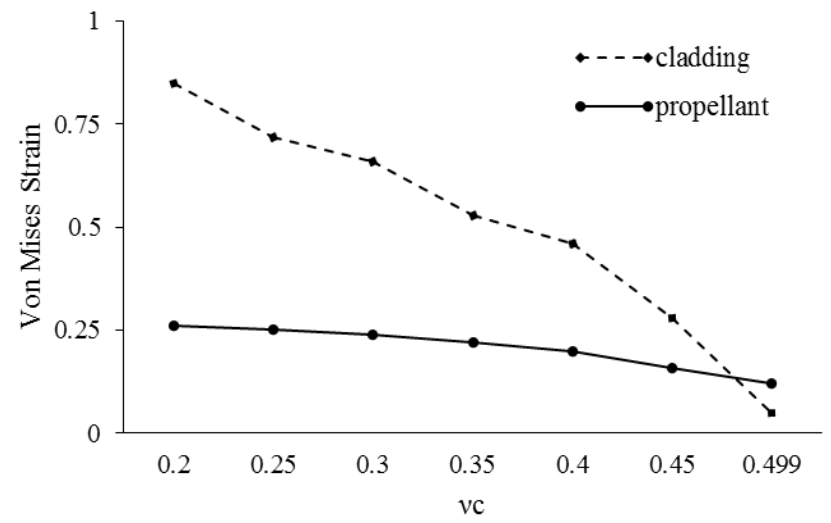

Figure 5. The Von Mises strain of dangerous point variation with the cladding poisson's ratio.

\section{Conclusions}

In this paper, by using the control variable method and finite element method, the Von Mises strain of the solid rocket motor grain's dangerous point in different situations have been compared under the internal pressure. The variables are the initial elastic modulus of propellant, the poisson's ratio of propellant, the initial elastic modulus and poisson's ratio of cladding. In order to analysis the rules of Von Mises strain of dangerous point variation with these variables, four test groups are performed. Each test group only had one variable, while the other three were fixed. Through changing the value of variable, the relationship between the Von Mises strain of dangerous point and variables are obtained.

(1) Von Mises strain of dangerous point growth soon with the decrease of propellant's elastic modulus. And for the propellant with low modulus, internal pressure is a major cause that affects its structural integrity.

(2) The effect of poisson's ratio of propellant is very obvious, which is much bigger than the influence of its initial modulus.

(3) The influence of cladding's initial modulus is small. when the initial modulus of cladding changes for 100 times, the change of the value of grain Von Mises strain is just 7.6\%

(4) Along with the increase of cladding poisson's ratio, dangerous point Von Mises strain of the propellant and cladding are decreasing. But the influence of cladding poisson's ratio on cladding is greater than its influence on propellant. 
In a word, as the main component bearing the load, the propellant plays an important role in the structure integrity of the motor gain. On the premise of considering other performance, the propellant initial modulus should be appropriate to improve and the cladding poisson's ratio cannot be too small.

\section{References}

[1] Z.B. Liu, F.X. Li, Y.S. Li, et al. Deformation of solid propellant grains under axial acceleration load, J. Propul. Tech., 25, 2 (2004)

[2] S.R. Kong, G.Q. Xing, Z.Y. Zhang. Effect analysis of geometric parameters on the structural integrity of wheel-shaped grain in solidified temperature-reducing process, Aero Weaponry, 44, $3(2011)$

[3] B. Sun, Z. Liu, Y.X. Duo, et al. Strain field analysis of solid rocket motor grain with impurity, J. Solid Rocket Tech., 39, 2 (2016)

[4] Y.Q. Ding, F.Q. Lan. Material parameters influence on grain structure integrity under temperature load, Aero Weaponry, 40, 5 (2007)

[5] H. Liu, X.C. Li, J.H. Feng. An analysis of structure integrity of solid propellant grain under vibration overload, Tactical Missile Tech., 30, 5 (2009)

[6] Z.C. Zhu, E. Cai. A finite element analysis of three dimensional temperature and stress fields for solid rocket motor grain, J. Propul. Tech., 18 (1997)

[7] T.Q. Yang, W.B. Luo, P. Xu, et al. Viscoelastic theory and application (Science Press, Beijing, 2004) 